Sharif University of Technology
Scientia Iranica
SCIENTIA
IRAN Transactions B: Mechanical Engineering
www.scientiairanica.com

\title{
Numerical study on the effect of the concave rigid boundaries on the cavitation intensity
}

\author{
M.T. Shervani-Tabar and R. Rouhollahi* \\ Faculty of Mechanical Engineering, University of Tabriz, Tabriz, Iran.
}

Received 27 June 2015; received in revised form 12 February 2016; accepted 2 July 2016

\section{KEYWORDS}

Cavitation bubble;

Boundary integral method;

Liquid jet;

Velocity vector;

Pressure contour;

Concavity.

\begin{abstract}
In this paper, we study the motion of a cavitation bubble near a concave boundary from a theoretical perspective. To illustrate the effect of the surface concavity of the boundary, boundary integral and finite difference methods are utilized to investigate the more detailed process of jet formation. Bubble shapes and its lifetime, movement of the bubble centroid, pressure contours, and velocity vectors are used to demonstrate the numerical results. The velocity of a liquid jet impacting on the far side of the bubble surface tends to increase with decreasing boundary concavity. This result suggests that higher pressures can occur when cavitation bubbles collapse near a concave boundary. With the increase of boundary concavity, the time evolutions of the bubble growth and collapse tend to increase, and the liquid jet is formed later.
\end{abstract}

(C) 2017 Sharif University of Technology. All rights reserved.

\section{Introduction}

Cavitation is one of the major problems that leads to a severe reduction in operating efficiency of the turbo pumps. The interaction between the bubble and boundaries is of great importance in the study of cavitation damage due to the bubble collapsing near a rigid boundary [1]. When a comparable-sized bubble is located near a rigid boundary, the bubble motion is significantly influenced by the surface curvature of the boundary, which is characterized by parameter $\xi$, giving convex walls for $\xi<0$, concave walls for $\xi>0$, and a flat wall when $\xi=0$. The geometry of a boundary surface is another important factor influencing the damage of material [2]. Cavitation phenomenon has undesirable consequences for turbo pumps such as extraordinary noise, vibration, blades corrosion, and efficiency reduction [3]. All liquids evaporate at certain pressure (vapor pressure) and

*. Corresponding author. Tel.: +984134265062;

E-mail addresses: msherv@tabrizu.ac.ir (M.T.

Shervani-Tabar); r.rooh2650@tabrizu.ac.ir (R. Rouhollahi) temperature. If the pressure is reduced to a pressure lower than the vapor pressure, the liquid starts to evaporate [4]. This is the first part of the cavitation due to the pressure drop at the inlet of pumps forming the vapor bubbles. These bubbles will move along with the flow to the other parts with higher pressure. If the pressure at the new location is high enough, these vapor bubbles collapse. In this case, cavities are formed in the liquid, causing other liquid particles to deviate from their paths and hit other surfaces like impeller blades with a very high impact speed. In these locations on the surfaces, depending on the severity of the impact, erosion occurs on the blades and the surface becomes porous. The growth and collapse of these vapor bubbles is called cavitation. Even though the simple bursting of bubbles is not much important, their intensity and repetition cause the released energy levels to become more than the yield strength of blade material. In this stage, blade disintegration and formation of small holes start [5]. Experimental studies show that the roughness of the surface due to the cavitation bubble collapse leads to intensifying the cavitation damage power. The roughness of a surface can generate local low-pressure 
regions facilitating the cavitation. Hence, it can be concluded that when the material losses its surface finish, the flow field around the cavitation bubbles is influenced by a new generated surface geometry. The velocity field and pressure distribution around the cavitation bubble near rigid surfaces are investigated numerically in many studies.

Shervani-Tabar et al. studied the velocity and pressure fields around the cavitation bubble during splitting and necking phases near a flat rigid surface [6,7]. The dynamic behavior of the gas bubble near rigid walls and its interaction with a set of flat rigid surfaces are investigated by the studies of Zhang et al. [8]. Zhao et al. considered oscillation of a cavitation bubble near a rigid surface experimentally and specified the maximum radius of bubble and collapse time based on procedures of bubble trembling [9]. Tomita et al. studied the growth and collapse of the cavitation bubble near a curved rigid surface. They showed that the surface convexity decreases the bubble lifetime; with increment of the surface concavity, the bubble lifetime increases [2]. Shervani-Tabar and Haji-Tabar investigated the dynamic behavior of the cavitation bubble near a curved rigid surface. In this study, bubble volume, pressure inside the bubble, movement of the bubble centroid, and the velocity of liquid jet near curved rigid boundaries are evaluated [10].

In the present paper, the behaviors of a cavitation bubble near the concave rigid boundary and the velocity and the pressure distribution around the cavitation bubble are studied numerically during the collapse phase. The boundary-integral and finitedifference methods are used for the numerical solution of the problem. The equations are written in both Lagrangian and Eulerian forms. This means that the relations at the points on the moving bubble are written in Lagrangian form and the relations at the points in the fluid are written in Eulerian form. Although the situation considered here is restricted to axisymmetric motion without mean flow, this result suggests that higher pressures can occur when cavitation bubbles collapse near a non-flat boundary.

\section{Explanation of model}

Consider a spherical bubble of initial radius, $R_{0}$, located above a rigid boundary. The fluid domain and the fluid boundary, including the bubble surface and rigid boundary, are labeled as $\Omega$ and $S$, respectively. The fluid domain around the bubble is assumed to be incompressible, inviscid with the irrotational motion. Also, surface tension is neglected (normally unimportant). According to the above assumptions, the behavior of the liquid flow around the cavitation bubble can be described in terms of velocity potential $\phi(r, z, t)$ satisfying the Laplace's equation:

$$
\nabla^{2} \phi=0
$$

Green's integral formula can be written for any sufficiently smooth function $\phi$ which satisfies Laplace's equation in domain $\Omega$ having piecewise smooth surface $S:$

$$
\begin{aligned}
C(p) \phi(p) & +\int_{s} \phi(q) \frac{1}{|p-q|} d S \\
& =\int_{s} \frac{\partial}{\partial n}(\phi(q)) \frac{1}{|p-q|} d S,
\end{aligned}
$$

where $p \in \Omega+S, q \in S$, and $\frac{\partial}{\partial n}$ is the normal derivative outward from $S$, and:

$$
C(p)= \begin{cases}4 \pi, & p \in \Omega \\ 2 \pi, & p \in S\end{cases}
$$

where $p$ is on $S$. We have an equation for either $\phi$ or $\frac{\partial \phi}{\partial n}$ on $S$ if the other is specified. Once both are known on $S$, Eq. (2) can be used to generate $\phi$ at any point $p \in \Omega$. The problem is axisymmetric and the centroid of the bubble is on the vertical axis, and the radial axis is situated on the rigid boundary.

When a bubble oscillates near a rigid boundary, its motion is spherical at initial stages of the growth phase; so, the radial motion of the bubble from zero volume to its initial volume can be calculated by Rayleigh-Plesset equation as follows:

$$
R \ddot{R}+\frac{3}{2} \dot{R}^{2}+\frac{P_{\infty}-P_{b}}{\rho}=0,
$$

where $P_{b}$ is the variable pressure inside the bubble, $P_{\infty}$ is the pressure in the far field, and $R$ is the radius of the bubble. An over-dot denotes differentiation with respect to time. The pressure inside the bubble consists of non-condensable gases and a constant pressure vapor. The non-condensable gases are assumed to be an ideal gas. Thus, the pressure inside the bubble is given by:

$$
P_{b}=P_{c}+P_{0}\left(\frac{\forall_{0}}{\forall}\right)^{k},
$$

where $P_{b}$ is the pressure inside the bubble, $P_{c}$ is the constant vapor pressure, $P_{0}$ is the initial pressure of non-condensable gases, $\forall_{0}$ is the initial volume of the bubble, and $k$ is the poly-tropic constant.

In order to keep the generality of the problem, all the variables have been normalized by using the bubble's maximum radius $R_{m}, \Delta p=p_{\infty}-p_{c}, \sqrt{\Delta P / \rho}$ and $R_{m} / \sqrt{\Delta P / \rho}$, respectively, similar to the characteristic length, pressure, velocity, and time. Thus, the 
dimensionless variables are yielded as follows:

$$
\begin{gathered}
\left\{R^{\prime}, \gamma, t^{\prime}, \phi^{\prime}, \psi^{\prime}, \varepsilon, \delta\right\}=\left\{\frac{R}{R_{m}}, \frac{H}{R_{m}}, \frac{t}{R_{m}} \sqrt{\frac{\Delta P}{\rho}}\right. \\
\left.\frac{\phi}{R_{m}} \sqrt{\frac{\rho}{\Delta P}}, \frac{\psi}{R_{m}} \sqrt{\frac{\rho}{\Delta P}}, \frac{P_{0}}{\Delta P}, \sqrt{\frac{\rho g R_{m}}{\Delta P}}\right\}
\end{gathered}
$$

where $\gamma, \varepsilon$, and $\delta$ are the stand-off parameter, the strength of the initial pressure inside the bubble, and the buoyancy effect, respectively. Therefore, dimensionless form of Rayleigh-Plesset equation is as follows:

$$
R \ddot{R}+\frac{3}{2} \dot{R}^{2}+1-\varepsilon\left(\frac{R_{0}}{R}\right)^{3 \kappa}=0 .
$$

This equation can be solved by Newton-Raphson iterative method for different values of $\varepsilon$. In this work, $\kappa$ is taken to be 1.4. Now, If $\varepsilon$ is taken to be 10 , then $R_{0}$ is obtained 0.3804 .

As liquid particles remain on the surface of the bubble, the velocity of the bubble surface may equate to the liquid particle velocity. Thus, the moving boundaries' location can be updated in a Lagrangian manner according to (kinematic boundary condition):

$$
\frac{D X}{D t}=\nabla \phi, \quad X \in S
$$

In order to obtain the rate of change of velocity potential on the bubble surface, the unsteady Bernoulli equation is used for the moving boundaries (the dynamic boundary condition):

$$
\frac{D \phi}{D t}=\frac{P_{\infty}-P_{b}}{\rho}+\frac{1}{2}|\nabla \phi|^{2}-g(z-H) .
$$

The governing equation with dimensionless variables is given as follows:

$$
\begin{aligned}
& \frac{D X^{\prime}}{D t^{\prime}}=\nabla \phi^{\prime} \\
& \frac{D \phi^{\prime}}{D t^{\prime}}=1-\varepsilon\left(\frac{R_{0}}{R}\right)^{3 \kappa}+\frac{1}{2}\left|\nabla \phi^{\prime}\right|^{2}-\delta^{2}\left(z^{\prime}-\gamma\right) .
\end{aligned}
$$

To solve Eq. (10) to obtain the time evolution of $\phi^{\prime}$, we require initial conditions. To do so, for a very small spherical Rayleigh bubble of radius $R_{0}$, we suppose that the velocity potential is zero on the bubble surface at the initial time. According to Best [11], the vapor bubble generated due to a high local energy input in its initial minimum volume contains a high pressure mixture of gas and vapor and has a spherical shape. The magnitude of the velocity potential, and consequently the magnitude of the bubble boundary in its initial minimum spherical shape are equal zero. But, due to a very high difference between the pressure of the bubble contents and the pressure in the far field, the bubble surface at its initial minimum volume has to be accelerated. It should be noted that by growing of the vapor bubble, the pressure inside the bubble decreases. Therefore, by decreasing the pressure inside the bubble, the pressure difference between the bubble contents and the far field, which gives out the bubble motion, decreases, and consequently results in the bubble collapse after the bubble reaches its maximum volume. At this time, the normal velocity potential is also zero on the rigid wall.

\section{Numerical solution strategy}

In the present study, half of the rigid boundary is discretized by $N$ linear elements, while half of the bubble surface is discretized by $M$ cubic spline segments, as shown in Figure 1.

Elemental values $\left(\phi\right.$ and $\left.\frac{\partial \phi}{\partial n}\right)$ are approximated with constant functions defined by their values at the collocation points, located at the midpoint of the elements. The boundary-integral-equation method is applied to obtain a numerical solution to the problem. Given an initial velocity potential of the bubble moving boundary and its normal derivative on the rigid boundary, $M+N$ elemental values are supplied. Therefore, in case $p_{i}$ is located on the boundary of the liquid domain, the discretization of Eq. (2) can be written as follows:

$$
\begin{aligned}
2 \pi \phi\left(p_{i}\right) & +\sum_{j=1}^{M+N}\left\{\phi\left(q_{i}\right) \int_{s_{j}} \frac{\partial}{\partial n}\left(\frac{1}{\left|p_{i}-q_{j}\right|}\right) d S\right\} \\
& =\sum_{j=1}^{M+N}\left\{\frac{\partial}{\partial n}\left(\phi\left(q_{i}\right)\right) \int_{S_{j}} \frac{1}{\left|p_{i}-q_{j}\right|} d S\right\} .
\end{aligned}
$$

After substituting the known values, the boundary integral equation will be simplified to a linear equation system as follows:

$$
A \phi=B \frac{\partial \phi}{\partial n}
$$

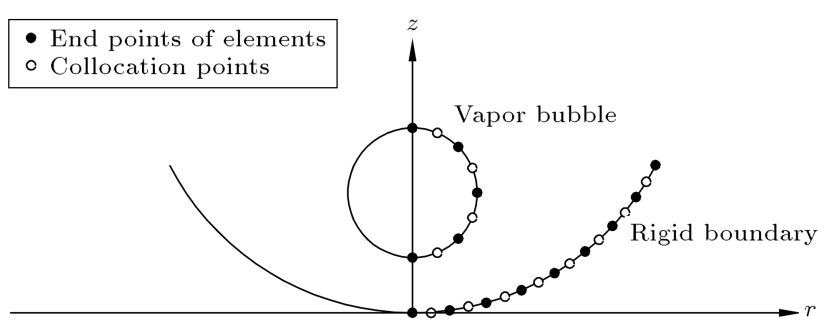

Figure 1. Discretization of the bubble boundary and the interface of liquid domain with the rigid boundary. 
We solved this linear equation system by Gaussian elimination method and obtained the normal velocity on the bubble surface and the velocity potential on the rigid boundary. The velocity potential on the bubble surface is determined by the Lagrangian form of the unsteady Bernoulli equation at the next time step as follows:

$$
\begin{aligned}
& \phi_{i}(t+\Delta t)=\phi_{i}(t) \\
& \quad+\Delta t\left\{\frac{P_{\infty}-P_{b}}{\rho}+\frac{1}{2}|\nabla \phi|^{2}-\delta^{2}(z-\gamma)\right\},
\end{aligned}
$$

where $\Delta t$ is an adaptive (variable) time step for capturing the bubble behavior better during the last stages of its collapse phase to avoid the instability problem. The value of $\Delta t$ which is used for the numerical integration of Eq. (11) to update the moving boundaries and their velocity potential can be obtained by:

$$
\begin{aligned}
\Delta t & =\frac{\Delta \phi}{\frac{D \phi}{D t_{\max }}} \\
& =\frac{\Delta \phi}{\left.\max \left|1+\frac{1}{2}\right| u_{\max }\right|^{2}-\delta^{2}(z-\gamma)+\varepsilon\left(\frac{R_{0}}{R}\right)^{3 \kappa} \mid},
\end{aligned}
$$

where $\Delta \phi$ is the maximum increase in the velocity potential on the moving boundaries between two consecutive time steps and is taken to be 0.05 in the present work.

Once the shape of the bubble is known, it is possible to compute the velocity and pressure fields in the liquid domain outside the bubble. So, the liquid domain is discretized by evenly spaced fixed points. It is noteworthy that during the growth phase of the bubble, some nodes in the neighborhood of the bubble boundary will be located inside the bubble, but during the collapse phase of the bubble, some nodes will be added into the liquid domain. Therefore, the nodes must be reordered in every time step to use the nodes outside the bubble and inside the liquid domain for simulation of the problem, and exclude the nodes located outside the liquid domain from the computation area.

We can obtain the velocity potential at any point inside the liquid domain on its four satellite points (see Figure 2) by having the velocity potential at the collocation points on the bubble boundary and rigid wall by using boundary integral equations.

The vertical and radial components of the velocity of each fixed point inside the liquid domain can be obtained by employing a central finite-differencing scheme:

$$
U=\frac{\phi_{r}(i, j)-\phi_{l}(i, j)}{R_{r}(i, j)-R_{l}(i, j)}
$$

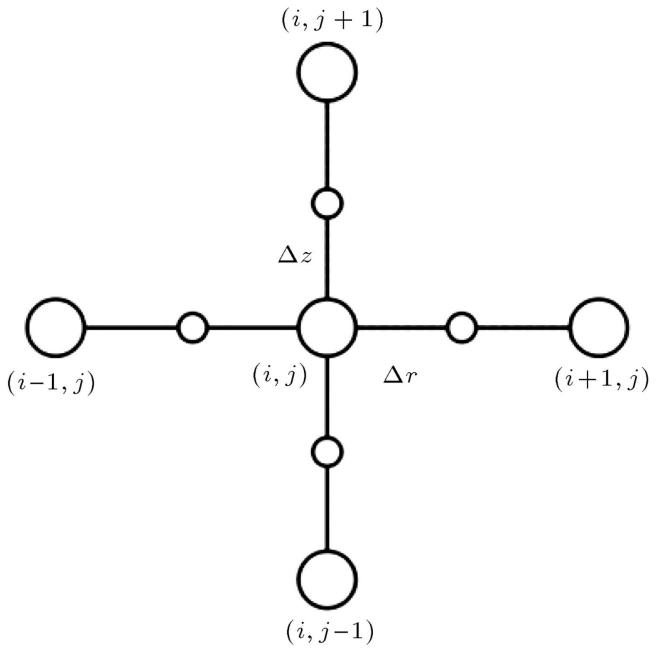

Figure 2. Fixed point inside the liquid domain and its four satellite points.

$$
V=\frac{\phi_{u}(i, j)-\phi_{d}(i, j)}{Z_{u}(i, j)-Z_{d}(i, j)},
$$

where $i$ and $j$ specify the coordinates of each fixed point; $U$ and $V$ are the horizontal and normal velocities of the specified point, respectively; $\phi_{r}$ and $\phi_{l}$ are the potential velocities on the right- and left-hand side neighbors of each fixed point, respectively; and $\phi_{u}$ and $\phi_{d}$ are the potential at the satellite points located above and below each fixed point, respectively.

With the value of the potential velocity at the fixed points inside the liquid domain, the pressure field around the bubble can be obtained by the unsteady Bernoulli equation as follows:

$$
\begin{aligned}
& P=P_{\infty}-\left(\frac{\partial \phi}{\partial t}+\frac{1}{2}|\Delta \phi|^{2}-\delta^{2}(Z-\gamma)\right)\left(P_{\infty}-P_{c}\right), \\
& |\nabla \phi|^{2}=U^{2}+V^{2}
\end{aligned}
$$

The computations will be continued until the liquid jet becomes fully-developed and reaches the opposite side of the bubble surface.

\section{Results and discussion}

The evolution of a bubble and dynamic behavior of the liquid around the bubble is studied under a definite arbitrary condition. The liquid domain is discretized into 2500 nodes. Table 1 summarizes the parameters used for the numerical simulation in this paper.

The calculations provide insight into the physics of fluid behavior by calculating bubble shape, shape and speed of liquid jets, velocity vectors, pressure fields, and the sites of intense pressure in particular that could lead to mechanical defects.

As shown in Figure 3, the bubble grows nearly spherically until it reaches a maximum volume, and 
Table 1. Table of physical properties and problem data.

\begin{tabular}{clc}
\hline$\rho$ & Density of the liquid & $1000 \mathrm{~kg} / \mathrm{m}^{3}$ \\
$P_{\infty}$ & Pressure in the far field & $100 \mathrm{kPa}$ \\
$P_{c}$ & Vapor pressure & $2 \mathrm{kPa}$ \\
$R_{m}$ & Maximum radius of bubble & $0.08 \mathrm{~mm}$ \\
$R_{0}$ & Initial radius of bubble & 0.3804 \\
$\delta$ & Buoyancy parameter & 0.0282 \\
$\gamma$ & Stand-of parameter & 1 \\
$\varepsilon$ & Strength parameter & 10 \\
$\Delta \phi$ & Maximum increase in potential & 0.05 \\
$M$ & Number of cubic spline element on half of bubble surface & 18 \\
$N$ & Number of linear element on half of rigid boundary & 60 \\
$\Delta r=\Delta z$ & Distance between main node and satellite node & 60 \\
$\Delta R=\Delta Z$ & Distance between main nodes & 0.05 \\
$\xi$ & Rigid boundary curvature & $0.4,0.8,1.2$ \\
\hline
\end{tabular}
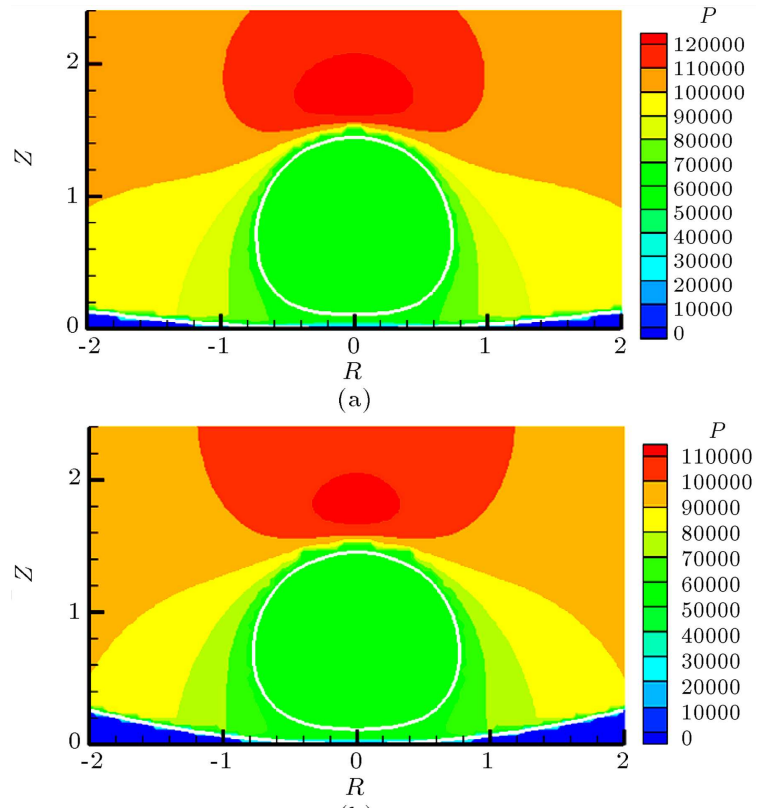

(b)

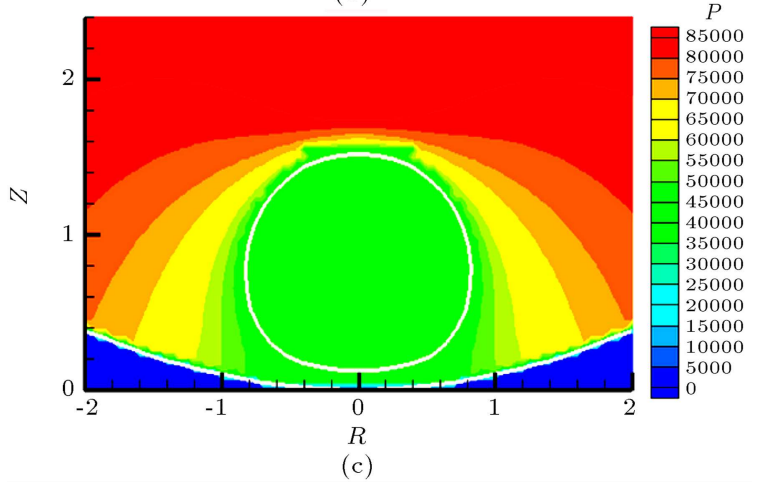

Figure 3. Profile of the bubble near the rigid boundary and pressure contours $(\mathrm{Pa})$ around cavitation bubble with $\gamma=1, \delta=0.0282$, and $\varepsilon=10$ at the jet formation moment: (a) $\xi=0.4$, time $T=2.2544$, (b) $\xi=0.8$, time $T=2.3073$, and (c) $\xi=1.2$ time $T=2.3429$.

then collapses. It is seen that when the bubble reaches its maximum, the pressure inside the bubble is lower than the pressure outside it and the pressure in the liquid domain is increased by going far away from the bubble. It should be said that the bubble grows until all of its kinetic energy has been imparted to the fluid. During the collapse phase of the bubble, a liquid jet develops on the far side of the bubble from the rigid surface and is directed towards it, and the lower part of the bubble is flattened and takes the shape of the rigid boundary. It should be noted that two main forces influence the bubble behavior above a rigid wall. The first is the buoyancy force exerted by the liquid, and the second is the Bjerknes attraction force exerted by the rigid surface. The buoyancy force causes the bubble to migrate away from the rigid surface, and the Bjerknes force attracts the bubble towards the rigid surface. The influence of the buoyancy force is shown by non-dimensional parameter $\delta$. When the buoyancy force is negligible in comparison with Bjerknes force, this case can happen.

The pressure and velocity fields around the bubble are also shown in Figure 4. At the last stages of the bubble collapse, a maximum high-pressure region is located above the liquid jet of the bubble, and the relative velocity of the liquid domain near the bubble jet is very high. In this case, there may be surface erosion due to the jet impact. Blake et al. [12] reported the results of pressure contours and velocity vectors around a laser-generated bubble near a flat rigid surface which also show the high pressure above the bubble and the high relative velocities in the jet. Features to note are the high pressure above the bubble before jet impact and the high relative velocities in the jet. It is interesting to note that in the final bubble collapse, a relatively broad liquid jet is formed by increasing the concavity. A close look at Figure 4 reveals that the magnitude of the maximum pressure above the liquid jet decreases with increasing the concavity of the rigid boundary, and the high pressure region is broadened. It may be the reason for the fact that by increasing the concavity of the rigid boundary, the liquid jet becomes broader. 

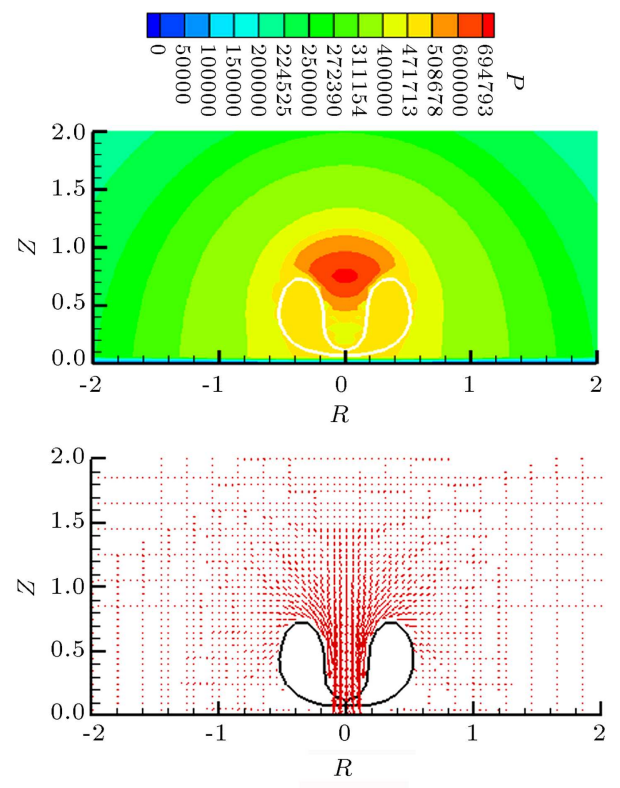

(a)
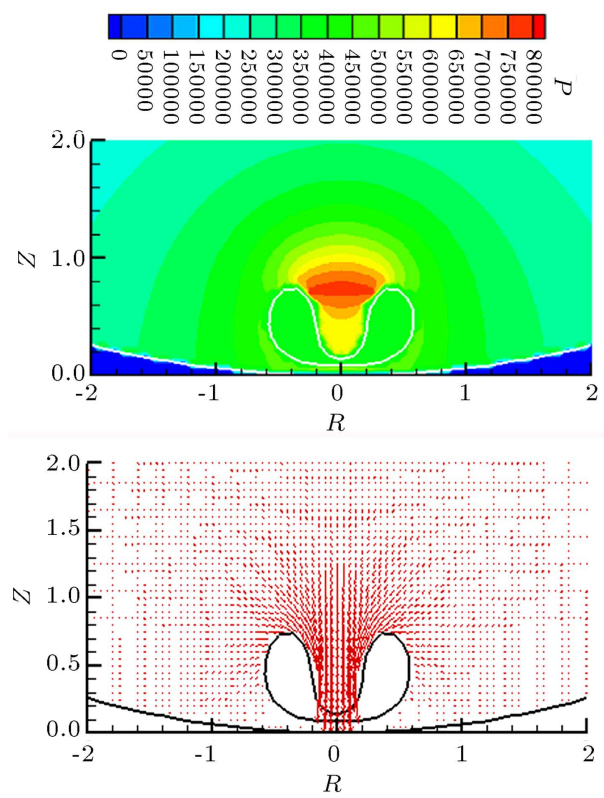

(c)
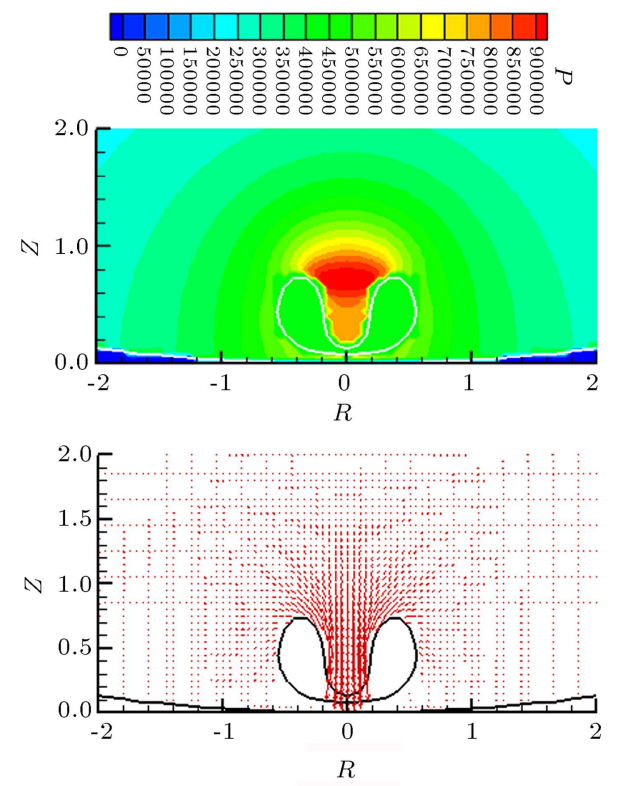

(b)
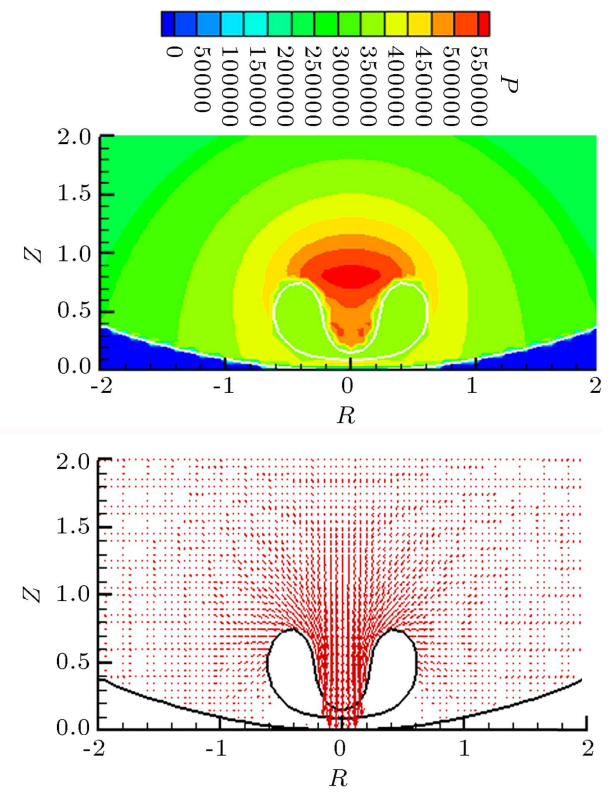

(d)

Figure 4. Velocity vectors and pressure contours $(\mathrm{Pa})$ around a cavitation bubble just prior to the bubble collapse with $\gamma=1, \delta=0.0282$, and $\varepsilon=10$ near flat, and three different concave rigid boundaries: (a) $\xi=0$, time $T=2.4971$, (b) $\xi=0.4$, time $T=2.5804$, (c) $\xi=0.8$, time $T=2.6817$, and (d) $\xi=1.2$ time $T=2.7854$.

Figure 5 shows the variation of the nondimensional volume of the bubble with respect to the non-dimensional time for different concavities. It is shown that the bubble expands until it reaches a maximum volume upon which it collapses; during the collapse phase, the volume of the bubble decreases. Also, it is observed that by increasing the concavity, the bubble volume increases in the final collapse phase.

The bubble centroid lies at the axis of symmetry. Figure 6 shows the movement of the bubble non-dimensional centroid with respect to the non- dimensional time. During a very short time in the late stage of the collapse, the translational motion of the bubble develops as a result of momentum conservation. In the growth phase, the bubble gets away from the boundary surface followed by a gradual attraction towards the boundary in the collapse process.

It is worth exploring the effect of the geometry of a boundary surface on the impact velocity of a liquid jet since it is regarded as one of the important factors in cavitation damage. Figure 7 shows the variation of the non-dimensional velocity of the liquid jet of the 


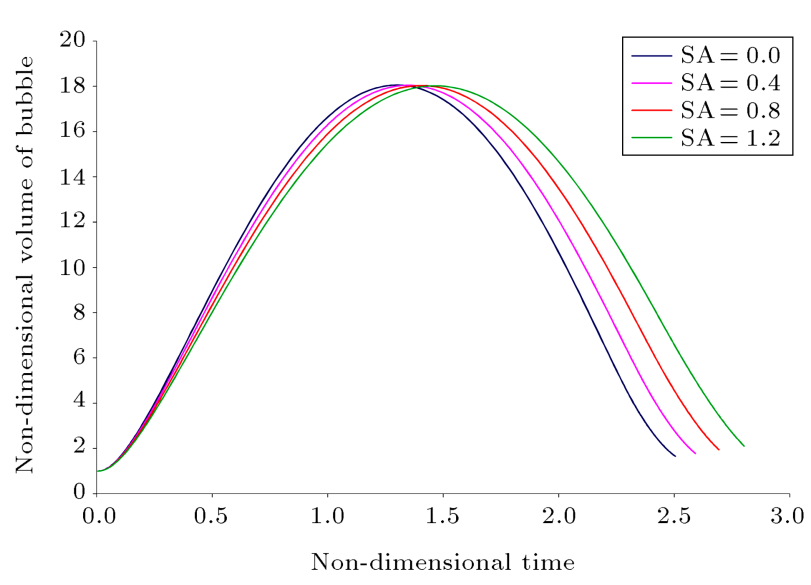

Figure 5. Non-dimensional volume of the bubble with respect to the non-dimensional time for different concavities and flat rigid boundary with $\gamma=1, \delta=0.0282$, and $\varepsilon=10$.

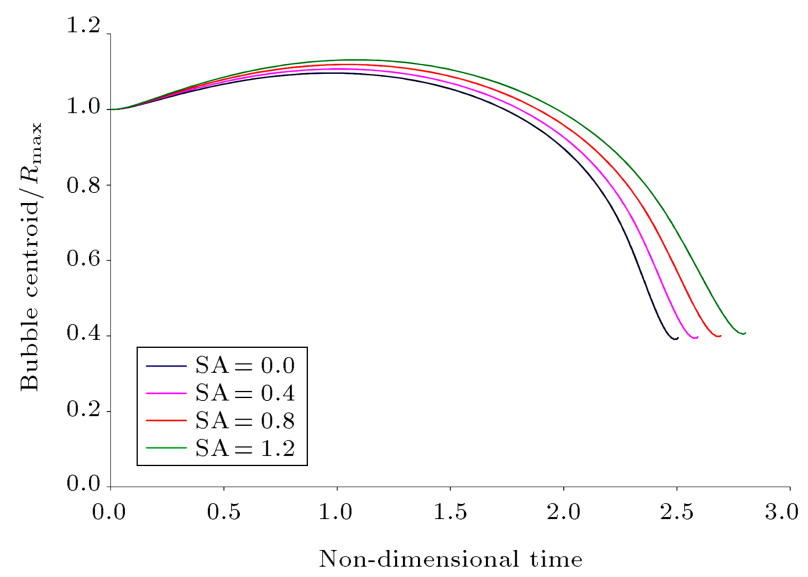

Figure 6. Movement of the bubble non-dimensional centroid with respect to the non-dimensional time for different concavities and flat rigid boundary with $\gamma=1$, $\delta=0.0282$, and $\varepsilon=10$.

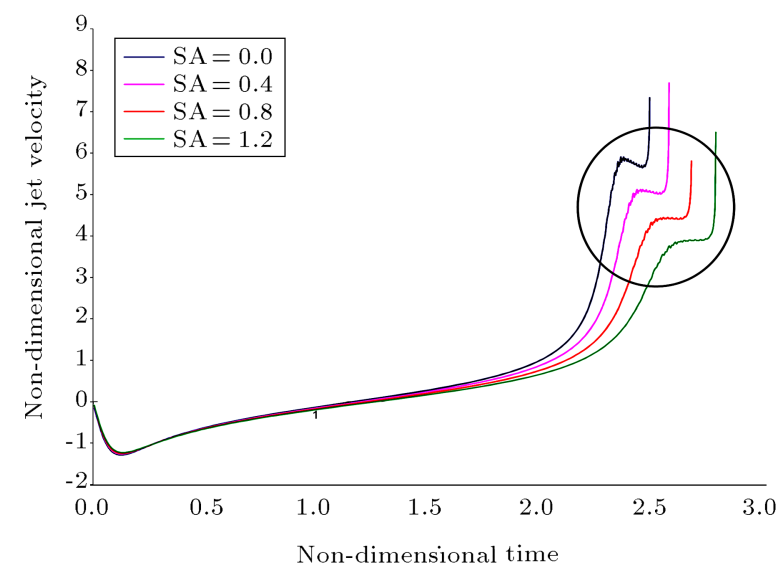

Figure 7. Non-dimensional velocity of the liquid jet with respect to the non-dimensional time for different concavities and flat rigid boundary with $\gamma=1, \delta=0.0282$, and $\varepsilon=10$.

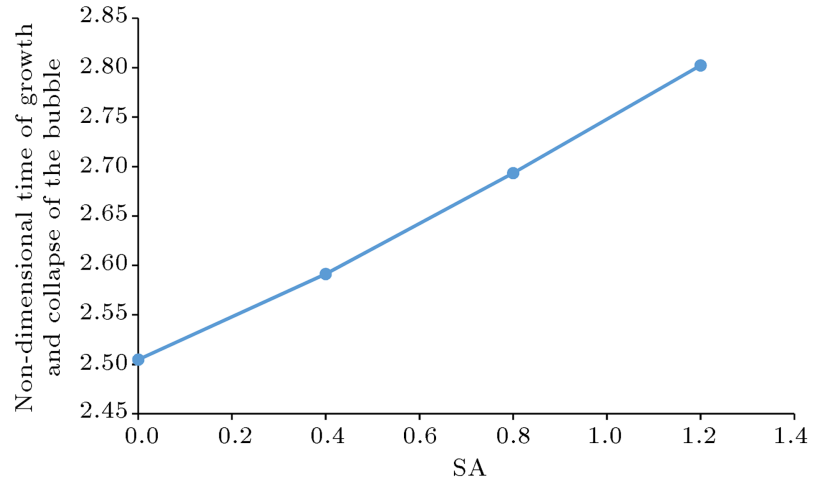

Figure 8. Non-dimensional time of growth and collapse of the bubble with respect to the rigid boundary concavity with $\gamma=1, \delta=0.0282$, and $\varepsilon=10$.

bubble at the axis of symmetry with respect to the non-dimensional time. It is shown that the bubble behaves approximately the same for all concavities during the growth phase by negative non-dimensional velocity. It is obvious that the velocity decreases very rapidly during the early growth phase. In the early collapse phase, increase of the jet velocity is very slow, but when jet is formed, the velocity increases very rapidly. During the collapse phase, the magnitude of the velocity of the liquid jet decreases when the concavity of the rigid surface increases. The area inside the dashed circle is the liquid jet formation period. It can be concluded that by increasing the concavity of the rigid boundary, the liquid jet is formed later and then the bubble collapses later.

Figure 8 shows the non-dimensional time of growth and collapse of the bubble with respect to the rigid boundary concavity. It is obvious that a concave wall makes the period of the bubble motion longer than that obtained in the flat boundary case with $\xi=0$ and $\gamma=1$. It is obvious that by increasing the period of the bubble, the destructive power of cavitation also increases.

\section{Conclusion remarks}

This study is particularly useful in predicting the interaction between the bubble and boundaries, which is of great importance in the study of cavitation damage due to the bubble collapsing near a rigid boundary. In this paper, cavitation bubble behavior, pressure distribution, and velocity field around the bubble near concave rigid boundaries have been investigated numerically during growth and collapse phases. The study is performed for three different concave rigid walls. Two methods are applied to gain general knowledge about bubble behavior near a concave rigid boundary: one utilizes the boundary integral method to obtain detailed features of bubble shape over the time, including liquid jet formation; the other is the finite 
differencing method which calculates velocity field and pressure distribution inside the liquid domain. Results show that the velocity of the liquid jet impacting on the boundary surface tends to increase with decreasing concavity. The bubble period becomes longer about the more concave boundary. When a boundary is more concave, the translational bubble motion is evident. It is interesting to note that the high pressure region above the liquid jet allocates more area to itself by increasing the concavity of the boundary, and this is the reason for the fact that by increasing the concavity of the rigid boundary, the liquid jet becomes broader. Results also show that the magnitude of the maximum pressure above the liquid jet decreases by increasing the concavity of the rigid boundary.

As a final result, when a boundary surface becomes rough due to exposure to cavitation for a long time, delayed formation of the liquid jet and increase of the bubble period by increasing the concavity of the rigid surface are firm reasons for increasing the cavitation damage power. Therefore, it is possible to prevent the advancement of the cavitation damages by periodic control of turbo-machineries blades.

\section{References}

1. Taib, B.B. "Boundary integral method applied to cavitation bubble dynamics", PhD Thesis, University of Wollongong, Australia (1985).

2. Tomita, Y., Robinson, P.B., Tong, R.P. and Blake, J.R. "Growth and collapse of cavitation bubbles near a curved rigid boundary", J. Fluid Mech., 466, pp. 259-283 (2002).

3. Laborde, R., Chantrel, P. and Mory, M. "Tip clearance and tip vortex cavitation in an axial flow pump", ASME J. Fluid, Eng., 119, pp. 680-685 (1997).

4. Nourbakhsh, A., Jaumote, A., Hirsch, Ch. and Parizi, H.B., Turbopumps and Pumping Systems, Springer, pp. 42-49 (2008).

5. Mackey, R., The Practical Pumping Handbook, pp. 5357 (2004).

6. Shervani-Tabar, M.T., Rezaee-Barmi, A. and Mahmoudi, S.M.S. "Velocity field and pressure distribution around two parts of a cavitation bubble after its splitting near a rigid boundary", Fifth International Symposium on Cavitation (CAV2003), Osaka, Japan (2003).

7. Shervani-Tabar, M.T., Rezaee-Barmi, A., Mahmoudi, S.M.S. "Velocity field and pressure distribution around a collapsing cavitation bubble near a rigid boundary during the necking phenomenon", Fifth International Symposium on Cavitation (CAV2003), Osaka, Japan (2003).

8. Zhang, A.M., Yao, X.L. and Feng, L.H. "The dynamic behavior of a gas bubble near a wall", Ocean Engineering, 36, pp. 295-305 (2009).

9. Zhao, R., Xu, R., Shen, Z., Lu, J. and Ni, X. "Experimental investigation of the collapse of lasergenerated cavitation bubbles near a solid boundary", Optics \& Laser Technology, 39, pp. 968-972 (2007).

10. Haji-Tabar, H. "Numerical investigation of the cavitation bubble growth and collapse in adjacent with the concave and convex curved rigid surfaces by Boundary Integral Equation Method (BIEM)", M.Sc. Thesis, Mechanical Engineering Faculty, University of Tabriz (1384).

11. Best, J.P. "The dynamics of underwater explosions", PhD Thesis, University of Wollongong, Australia (1991).

12. Blake, J.R., Pearson, A. and Otto, S.R. "Boundary integral methods for cavitation bubbles near boundaries", Cav2001:lecture.004 (2001).

\section{Biographies}

Mohammad-Taghi Shervani-Tabar received the PhD degree in Mechanical Engineering from University of Wollongong in 1995. He is currently a Professor in University of Tabriz. His main interests are in the area of cavitation and bubble dynamics, boundary element method, and computational fluid dynamics. His interest extends to the field of droplet management, fluid flow in porous media (oil recovery enhancement), and impinging jets. Professor Shervani-Tabar has published more than 45 publications.

Roya Rouhollahi earned her MSc degree in Mechanical Engineering from University of Tabriz in 2010. She is currently a Mechanical Engineering PhD student in University of Tabriz. The main focus of her thesis is Electro-Hydro-Dynamic (EHD) phenomena in Falling Film for cooling and enhancement of heat transfer in condensers and evaporators. She is a Teaching Assistant at University of Tabriz and Senior Research Engineer at Artasam Company in Tabriz. Her major fields of interest are cavitation and bubble dynamics, microfluidic, non-Newtonian fluids, and Electro-HydroDynamic (EHD) phenomenon. 